\title{
Jitter Model and Signal Processing Techniques for Pulse Width Modulation Optical Recording
}

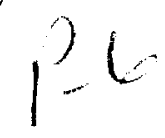

\author{
Max. M.-K. Liu \\ Department of Electrical and Computer Engineering \\ University of Arizona \\ Tucson. AZ 85721
}

\section{IBSTRACT}

Thus paper duscusses a lutur model and signai processing tecnMalues ior tata recovery in Pulse Widin Modulation I PWM ontiwi recordme LI PWM. intormation is stored througt modulating sizes of sequentual marks alternating in magnetic polarization or 11 matertai structure. Jitter. defined as the deviation from the "risinal mark size in the time domain. will result in crror detec- Ion if it is excessively large. This paper takes a new approach In data rccovery ny rirst using a high speed counter clock to convert tıme-marks to ampiltude-marks, and uses signai processine techniques to minimize jitter according to the jitter model. The ugnal processing techniques inciude motor speed and intersvmbol interierence equalization. differential and additive detection. and hiterential and auditive modulation

\section{Introduction}

Optical jata storage is built upon many disciphnes. Tlırough the applications of these disciplines. storage densities and access -peeds have been improved significantly [1]. [n addition to the results that have provided shorter wavelength laser diodes. lighter iptical heads. and better understanding of media. the use of signal processing techniques is also important in extracting information from noisy signals. As a result, this paper will study how data can he modulated. equalized, detected. and demodulated to achieve inigher density storage.

\section{Background}

Since the magneto-optical (M-O) and phase change media have inly two different polarizations or states. information can be stored only througn modulating marks alternating in polarization or state. In this paper. pulse width modulation (PIVM) is considered where marks of varıabie size according to the input signai are recorded.

[n detecting a readback signal, the peak detection technique is -ommoniy used i2][3]. That is, a signal of peaks at mark boundarles is first generaced by a differentiator if necessary. To recover the original signal, a time window is used to decide whether a peak falls into it or not. The time window is generally derıved irom the readback signal by a phase lock loop (PLL) [4].

\section{Approach of This Paper}

In this paper, a different approach is taken in recovering the original sıgnal. First, mark boundaries are detected by methods such as the peak detection method described above. In the second step. Instead of using the time window method, a high speed counter clock is used to quantize the mark size between two adjacent mark boundarles, as depicted in Figure 1. The counter output generates an integer number proportional to the time mark. In other words. this quantization step transforms the readback signal from time marks to amplitude marks, and signal processing lechniques are subsequently used.
There are two important awbantares it i..: mantization abproach. First. It converts a :lme-mark sienat to an amplis: :... nark signal. As a resuit of this conversion. ine joman nols : jitterl is at the same tume converted to amplutude romm nu. he which allows us to periorm signal processint in the mnonutg :?omain. Another advantage of this approacis is it proviries a tringue and integrated impiementation of varous euualization. setion. and demodutation algorithms This will become ciear in the subsequent discussion.

One disadvantage of this approach is the acded acuantization error in the quantuzation step. However it the lock is iast enouth. this quantization error is neqigible. For pxample, if the ork $\mathrm{s}$ pertod is one nsec and mark sizcs are mutiples of nsec. ne?

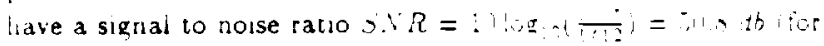
aiculation, see $[j]$ for example

Jitter, defined ${ }^{2}$ as the total deviation away trom the ontriat mark size by all possible causes. is the time domain nose. simiar to the ampitude noise in an amplitude modulated signai. There are vartous sources that contrioute to the total jitter. This paper will establish a jitter model that transtates all jilter sources into the total jitter, and study how the jitter can be reduced by irgal processing.

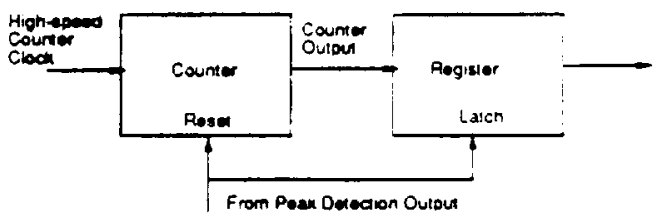

Figure 1: Quantization of Time Marks to Amplitude Marks

\section{Jitter Model}

This section describes a jitter model based on which equaiization. detection, and modulation techniques are described in the following sections. Although the model is not yet completely vertfied experimentally, it is formulated according to recent experiment and sımuiation studies $[i]-\{[\mid]$.

\footnotetext{
'If we asaume the disk linear velocity is $4 \mathrm{~m} / \mathrm{sec}$, this 100 nsec ume unic correapond to $400 \mathrm{~nm}$ mark unit. For a system using $(2.7)$ RLL code. the minimum mark aze is $1.2 \mathrm{sm}$. If we compare this with a typical diffraction limut at $1 \mathrm{um}$, this $100 \mathrm{nsec}$ is a reaconable aasumption.

2 In optical recording, since there are many sources causing marik size deviation. jitcer is deo defined differentiy. For example, in peaj detection, the viation. jitter is aleo defined differently. For example, in peax detection, the
deviation of mark boundary is called peak shift [3], and jitler is one of the causes due to random noise. In this paper. for simpiicitv. jitter is the tota result of all deviation causes, and each cause with be described in detail in the jitter model.
} 


\subsection{Whill Process Jitter Model}

In the write process moquiated mout lata io a dish drabe in a sequence of time marks . . $T,-1, T, T+1 . \ldots$ where $T$, is ine ime duration of mark; He can derine $t=\sum_{i=i}^{-i} T_{+}$io be the siarting ume of mark;

When the input signal is recoraid ontu a wisk, ibe spatal main

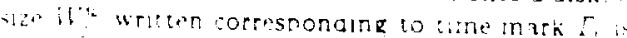

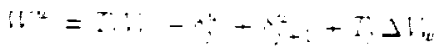

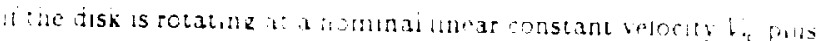

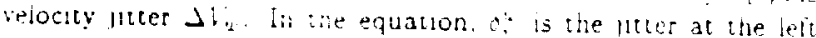
hand side of the mark. ${ }_{+}$: is at the right hand side. and fit is positive if it makes $i f$; smatler. Figure ?

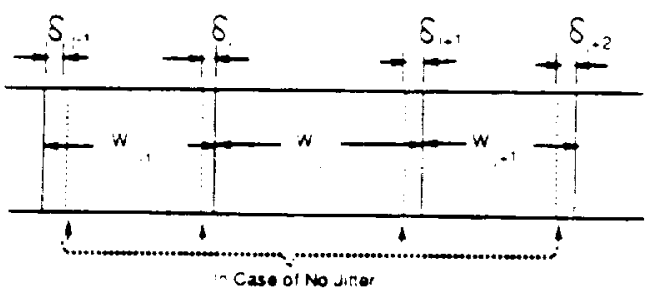

Figure 2: Mark Size anif issuchate! Jithor

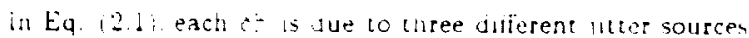

$$
\because=\dot{a},+\therefore+a,
$$

where

$f_{1, j}^{u}$ : Jitter due to random nolse such as electrontc noise and mag netic medum property fluctuation or defects. This first type of jitter is called notse jitter and otten simply called jittcr. This jitter has no correjauon with the input signal and is independent of adjacent jitter of the same kind.

$54:$ Jitter due to laser power and/or beam width Huctuation, or other simular mechanisms. This kind of jutter is called bit shift juter. This jutter is shutied in different direction at tho rising and falling euges of a mark in erase-and-write optical recording systems. where marks are "written" or "burned" only with even indexes. That is. the litter sequence $\left(\delta_{z}^{u}\right.$, $\because, \varepsilon_{2,+}, 1$ will be alternating in sign The magnituje of this fitter in general should be slowly varying and has stron! correlation with adjacent ones.

$i_{1}:$ Jitter due to intersymbol interierence (ISI). This jitter is a function $f\left(\ldots T_{1-1}, T_{2}, T_{1+1}, \ldots\right)$. of adjacent tume marks. This function can be linear or non-linear of ume mark sizes $\Gamma$, s. Physically. when a mark is being written. the longer the mark. the more heat is accumulated on the media, which results in this mark size dependent jitter.

\subsection{Read Process Jitter Model}

In the read process. the spatial mark size that is being read back can be simiarly expressed as

$$
i t_{j}^{r}=16^{2}-\delta_{j}^{r}+\delta_{j+1}^{r}
$$

where each jitter component has its similar counterpart described in the write process. Specifically
Appendix $N$

- lolse licter due to random recelver notst

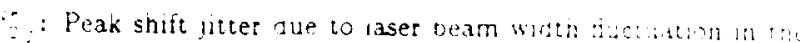
read process.

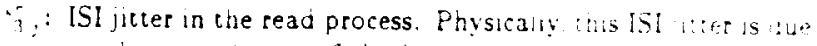
to the convolution of the iaser beam with whts a whllwh mark size. The tame mark read vut whi jetment on lie marn size recorded.

Including the motor speed jutter. we nawe $F=1 \% ?:$ -

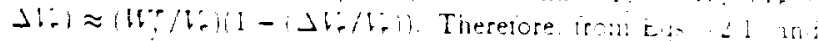
231 , we nave

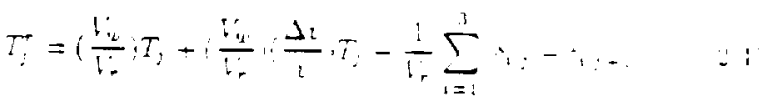

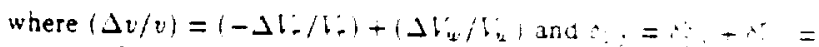

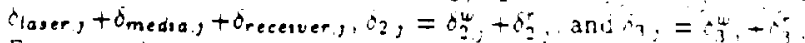

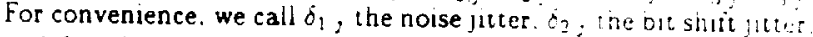
and $\delta_{3}$, the 1 SI jitter

\section{Equalization Techniques}

The objective of equalization discussed in :11/s sclion $1:$ cestore the original mark size $T$. from $T$ :

\section{(i). Write Velocity Jitter Correction}

The term $T_{1} \Delta V_{w}^{\prime}$ in Eq. $(2.1)$ is the jutlet causer of vejority Auctuation and is proportional to $T_{j}$. In tume-wincow-based peat detection systems where mark sizes are not quantized this $T$.proportional jitter is not important since $\mathrm{It}$ can be compensated easily by a phase lock loop (PLL) in the read process. However, when time marks are quantized and converted inco ampiltude marks, it is more difficult to use a PLL. In the following we explain how velocity fluctuation can be compensated by using the servo voltage in the disk drive.

A circuit that removes motor speed jitter is depicted in Fig 3 . In the figure, a voltage signal $v(t)$ that is proportional to the linear velocity of the disk with respect to the laser head is sent to an integrator. If the integracor is reset to zero at time $t$, the voltage output $U,(t,+1)$ of the integrator equals the spatial mark IV, at time $t_{j+1}$, and the comparator output changes irom " 0 " 10 "!". This transition will reset the integrator. change the $\mathrm{J} \mathrm{K}$ Fili. Flop state from on to off. and turn off the laser diolde Simiar operation repeats from tume $t_{j+1}$ to $t_{j+2}$

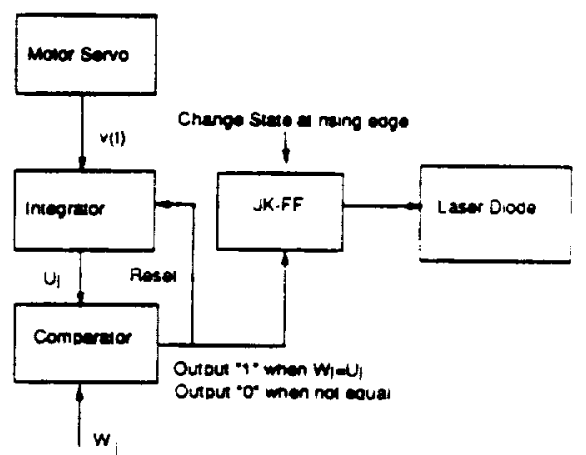

Figure 3: Circuit for Write Process Compensation 


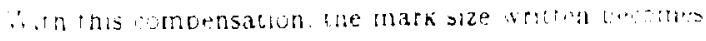

$$
\because:=13:-\because:-
$$

\section{ii). Read Process Equalization}

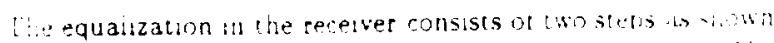

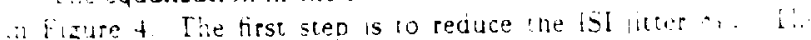
wat ij onduce lsi is to subtract each $r$ by an amounc ui $\because$ i:

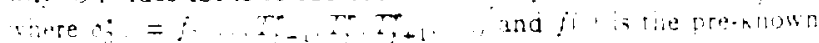

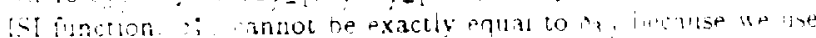
$\because$ instead of $F$ : 0 estumate the ISI nuter. Betcer esumation can be obtained by ising decision leedback equalization BFE

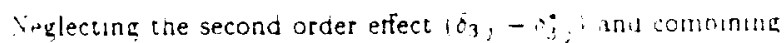
Equs. 2.1 and 3.1$\}$, we nave

$$
i_{i} i \approx \frac{\vdots}{i}, T_{1}\left(1-\frac{\Delta i}{i},-1 \frac{\vdots}{!}, \sum_{i=1}^{2} s_{i},-c_{1}-1\right)
$$

The second step is to correct the motor speed fitter in the read process. To accomplish this. we also use the motor servo valtage ignal to estimate the quanuty $\$ B / h$. based on which. We hite:

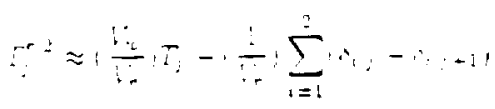

After these two steps, jitter that is left includes oniy $\mathrm{A}_{1}$; and $\therefore$. The first one is purely random and has no cortelation with idjacent ones. The second one is slowly varying and alternating in sign as a function of $j$. Instead of equalizing this second jitter directiy, the following detection techniques are used to make correct detection insensitive to this jitter

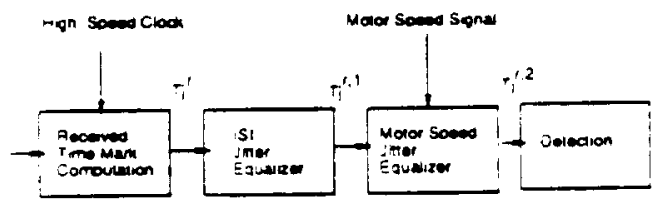

Eigure 4 Block Diagram in Recelver Equaiization ano Detection

\section{Detection Techniques}

Tile tirst method to recover the original mark size $\sigma_{;}$, from $\Gamma^{2}$ is cailed the Differential Interleaving Detection 1DID). If we subtract $T_{j+2}^{r 2}$ from $T_{:}^{r 2}$, the difference is:

$\Delta T_{j}=\left(T_{2}^{r, 2}-T_{j+2}^{r, 2}\right) \approx\left(\frac{V_{w}}{V_{j}}\right)\left(T_{2}-T_{j+2}\right)-\frac{1}{V_{p}}\left(\delta_{1, j}-\dot{\delta}_{1, j+2}-i_{1,+1}+\dot{\delta}_{1, j+3} j\right.$

where the term: $\delta_{2,}-\delta_{2,+2}-\delta_{2} ;+1+\delta_{2 ;+3}$ is of second order ind maybe neglected because of the strong positive corrciation between $\left(\delta_{2, j}, \delta_{2, j}+2\right)$ and $\left(\delta_{2, j+1}, \delta_{2, j}+3\right)$.

$\Delta T$, in Eq. (4.1) has only the random jitter. Comparing this with Eq. (3.3). this technique has a $3 \mathrm{db}$ power penaily. However. we may use maximum likelihood sequence detection (.ILSD) to letect each $\Delta T_{1}$ in a sequence of $\left(\ldots, \Delta \Gamma_{;}-2, \Delta T_{1}, \Delta T_{!}+2\right.$. to avoid this penaity. With this differential interleaving inetiod if the first two mark sizes $T_{1}$ and $T_{2}$ are predefined, subsequent $T$,'s can all be obtained from the differential terms.

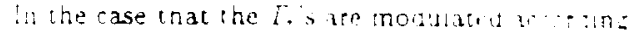

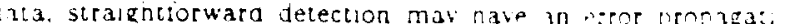
roblem. To solve this. a post-modutation me'sigj ated Li:

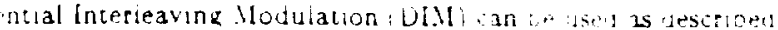
'it: next section.

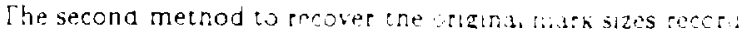
- ailed the Additue Interteaving Derpetion , MD This mer:. irst adds the two adlacent mark sizes reat Inat is:

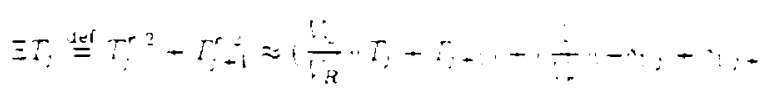

Irain, the term on, $-A_{2} ;+2$ is of serond orrer and negiected.

From the detected $1 \ldots \ldots \bar{Z}-i, \equiv \bar{Z} . \ldots+$. an all be recovered if the first $\Gamma_{1}$ is preqennes. This metro has the same error proparation probiem as blD It writen mark re modulated according to $\equiv T$, instead bi $F$ : art prontem completely removed. and this posi-morulation is inted Aditur incerleaving . Iodulation (AIM)

\section{Modulation Techniques}

For a given modulation coge that transiates an indut mossa into a sequence of mark sizes s; the oblertive at the prostmo: ulation DIM or AlM is to transiate $s$, into $\Gamma$, is inat at it. detection either $\Delta T$, or $\equiv T$, can be used directly to recover :n original $S$, without any error propagalion. Without ioss of gene: tiity, $V_{r}=V_{y}$ is assumed in the following discussion

\section{(i). Differential Interleaving Modulation}

For a given modulation code. assume eacn mark generate satisfies the following condition:

$$
0<S_{\min } \leq S_{1} \leq S_{\max }
$$

For DIM, we first define the initial vaiues $\Gamma_{1}$ and $\Gamma_{2}$, and suo sequent $T_{j}$ 's after $T_{1}$ and $T_{2}$ are obtained as tollows:

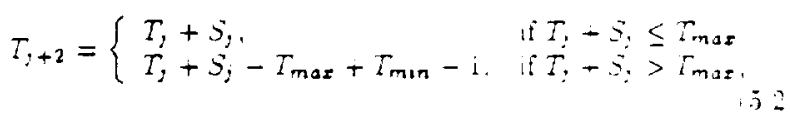

where $v<T_{\min } \leq T, \leq T_{\max }$ for eacn?

To make the modulation rule self-consistent when $T_{:}=T_{m n x}$ and $S_{j}=S_{\text {max }}$, from the second part of $E_{q} .521$. we need

$$
S_{\max }+T_{\min }-1 \leq T_{\max } \text { or } T_{\max }-T_{\operatorname{man}} \leq S_{\max }-1 . \quad 15.3
$$

By subtracting $T$, on both sides of $\mathrm{Eq} .(5.2)$, we have: $-\Delta T_{i}=T_{j+2}-T_{j}= \begin{cases}S_{j} & \text { if } T_{j}+S_{j} \leq T_{m} \\ S_{j}-T_{\max }+T_{\min }-1, & \text { if } T_{j}+S_{j}>T_{m 0}\end{cases}$

We note that if $T_{j}+S_{j} \leq T_{\max }$ is true in recording, $-\Delta T_{;}=$ $T_{j+2}-T_{j}=S_{j} \geq S_{\min }$, and if $T_{j}+S_{j}>T_{\max }$ is true in recording by Eq. $(5.3),-\Delta T_{j}=T_{j+2}-T_{j}=S_{j}-T_{\text {max }}+T_{\text {min }}-1 \leq 0$ Therefore:

$$
-\Delta T_{j}=T_{j+2}-T_{j}= \begin{cases}S_{j}, & \text { if }-\Delta T_{j} \geq S_{\min } \\ S_{j}-T_{\max }+T_{\min }-\mathrm{i}, & \text { if }-\Delta T_{j} \leq 0\end{cases}
$$

As a result, the differential pre-demodulation rule is: 


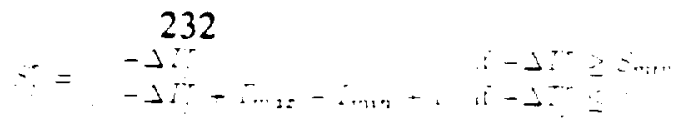

and the onginal 5 , can be recovered by simole threshoid detectum

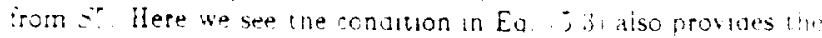

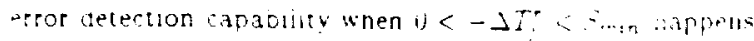

ii). Adelive Interieaving . Iodulation

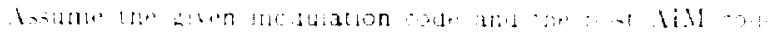

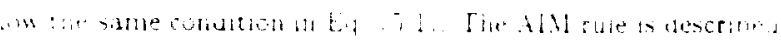

$$
\begin{aligned}
& \therefore-\therefore-i \cdot n
\end{aligned}
$$

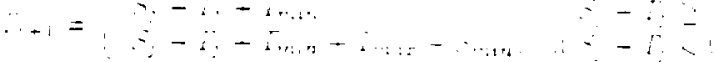$$
\therefore \text { insias }
$$

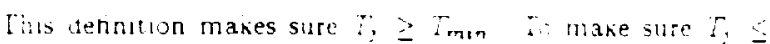
Mar we need the following wo adjutonal condutions substitut.

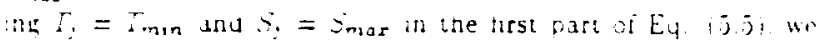
need

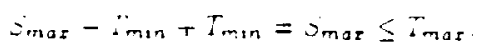

Substutulina $1 \therefore-T, 1$ with - i m the second part oi Eq. 55 , w. need

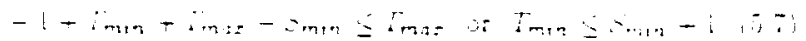

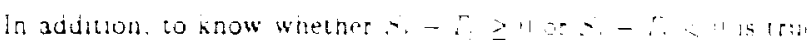

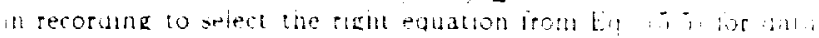

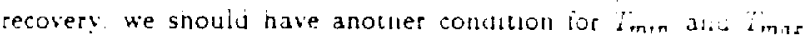
Sote that when $5:-T_{:}<0$ is true, we have: mul $T_{i}-T_{-1}=$

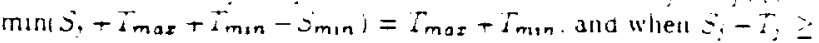

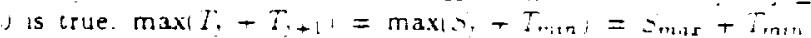
Theretore condition $(5,1)$ should be modified as:

$$
T_{\text {max }}>5 \min
$$

As a result. with the wo condituons 157 and 1.5 s. we can have the following All demodulation rull.

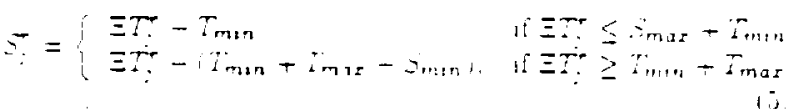

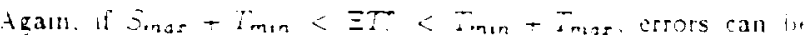
netected

\section{Simulation Examples}

Tins section exarnines how data recovery is amproved by unan the signa. processing teciniques discussed taritcr. To have a quantitative periormance evaluation. we use the mark error rate MERl as the criterion. which is detined as the ratio of the number of misdetected marks to the total numbet of input marks

In the following subsections, we first describe three different sets of the statistical parameters that are used in the computer simuiation programs. These three sets are used to describe three inicrent miter scenarios Vext, we describe nive differcit detection methods based on which MER is calcuiated. In smulation, cach $M E R$ is obtained at a grven signal to nolse ratio iSIR). where $S \times R$ is defined as

$$
S V R \equiv \frac{\text { rnit Mark Size }}{\text { R.IS Jitter ol } c}=\frac{(\Delta T) H_{r}}{\sqrt{\text { ianer }- \text { medin }-\sigma_{\text {recetver }}}}
$$

where $\Delta T$ is the unit of time. From this definttion. We see that SVR here onjy includes notse julter. At the end we will discuss the simulation resuits

\section{1 ppendix $N$ tatistical Parameters}

In simulation. time mark sizes are $\Delta T$. where $J T$. be $100 \mathrm{nsec}$. and $i$ is uniormiy distributed between in ant 5 isim. ilat to the (2.7) code but not exactiy). The disk . near worlt: set at $4.0 \mathrm{~m} / \mathrm{sec}$ in the both wrute and read process. For simpucit:

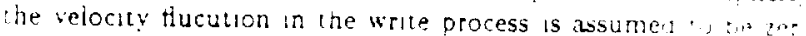
in all simulation cases lor assumine the velocity ontrolith ail : tone periectly. Three different sets of parameters are wited t cover the following scenarios:

1. All velocity jitter, bit shift jitter. and ISI ant: sinnifi. cant.

In this scenario, read velocity fuctuation range is si: $\quad \ldots \ldots+11$ : $\mathrm{m} / \mathrm{sec}$ of $10 \%$ of the mean velocity. Velocity is mantulum 1.0 i... slowiv varying within a ume mark period

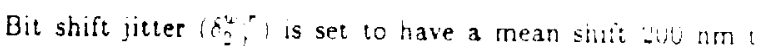
represent approxumately $20 \%$ of the laser beam width i. the diffraction limit is $(\mu \mathrm{m})$. and the fluctuation of : $(\mathrm{i}$ s sisti is $\pm 60 \mathrm{~nm}$. or approximately $\pm 6 \%$ of the laser beam witth The i, shift jitter is also maintained to be slowly varying in a thme marh period.

ISI jitter is assumed to be essentually a hinear humbum of $1 \%$ ume mark written. The proportional constant is "thom te be 0.05 in this scenario. The detection pertormall... . . Firure 5 .

\section{Only bit shift jitter is significant.}

In this scenario, velocity juter is set 10 zero. anil the ISi nuter is reduced from 0.05 in scenario 1 to U. 02. Other parameters are the same as those in scenario 1 . The detection prriormance in shown in Figure 6

\section{Only ISI is significant.}

In this scenario, velocity jitter is set to zero. and ine bit siriti jitter is reduced from $\pm 60 \mathrm{~nm}$ in scenario 1 to $=10 \mathrm{~mm}$ or $=$ of the laser beam width. Other parameters are mamiancd to the same as in scenario 1. The detection pertormance is show: Figure $i$

\subsection{Detection Methods}

Five detection methods are used in simulation. Thes ar

\section{Basic Detection.}

The basic detection method is based on thresioiv secection: and uses no equalization other than compensating the averare bu shift term $\overline{\delta_{2}}$. That is, tor each detected mark size. $T^{r}$, we suvtract $2 \overrightarrow{\delta_{2}} / V_{r}$ if $j$ is even and add the same amount if $j$ is odd.

\section{Basic Detection plus ISI Equalization.}

In addition to the average bit shift compensation. this second detection method equaiizes the ISI jitter.

\section{Basic Detection plus ISI and Velocity Equalization.}

In addition to the basic detection and ISI equalization. this detection method equalizes velocity fluctuation

\section{Differential Detection plus ISI and Velocity Equaliza-} tion.

This detection method equalizes ISI and velocity fluctuation but it does not cancel bit shift jittet in the rudimentary way of method 1. Instead. it uses the better differential detection method 


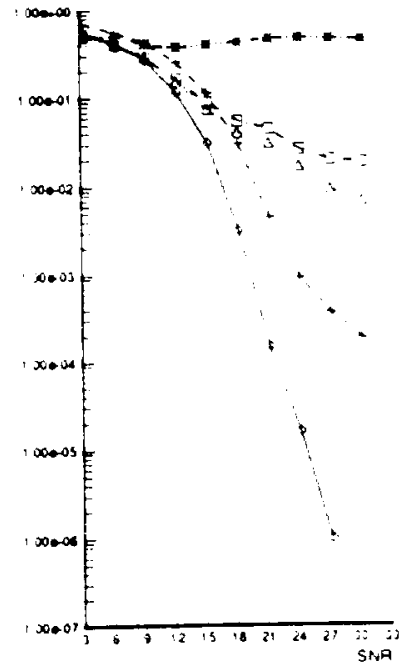

Figure 5: Mark Etror Rate of scenario I

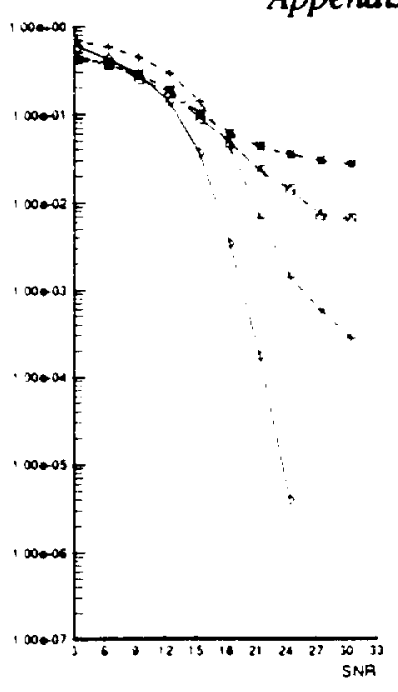

Figure o. Mark Error Rate of scenario:

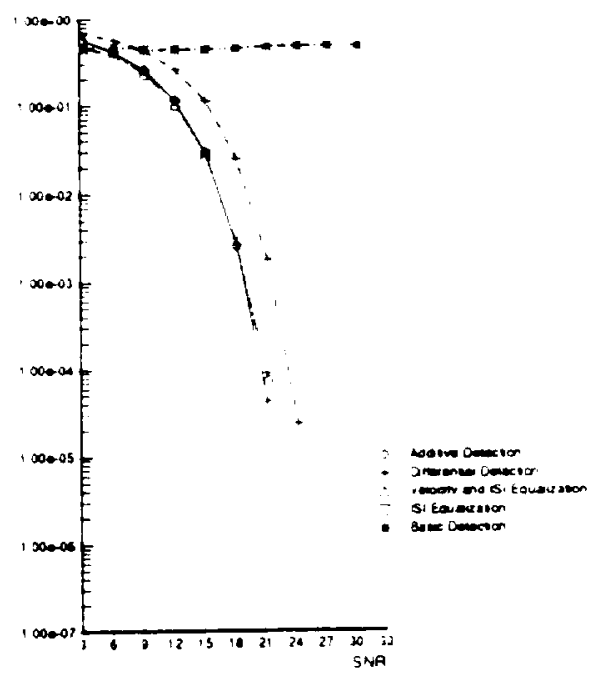

Figure 7 : Mark Error Rate of scrnark...

\section{(DID) 10 cancel the jitter}

5. Additive Detection plus ISI and Velocity Equlization.

This detection method is aimost the same as the last metho. except it uses additive detection rather than difierentiai detection described in section 4

\subsection{Discussion}

From the results, we may make the following observations.

1. The basic detection method is not an effective detection method if the nolse jitter is dominated by other jitter. As shown in Figures $j$ to $i$. MER can not be eflectively improved by increasing S.VR. This indicates the importance of equalization.

2. Methods 2 and 3 periorm almost the same if velocity lluc. tuation does not exist. These two methods perform equally as well as the DID and AID methods when gaussian noise dominates $(S N R<12$ ) (even better than DID).

3. Differential detection performs better than the first three detection methods. and is approximately $3 \mathrm{db}$ poorer than additive detection. This is what we predicled before. But interestingly, when SNR is greater than $25 \mathrm{db}$. DID reaches an error thoor if bit shift jitter is significant (see Figures 5.6 ). We do not see the similar foor when only ISI jitter is important (Figure 7). This error floor is likely due to the second order jitter effect that we have neglected. and this effect does not appear in the case of AID. When noise jitter dominates $(S N R<12$ in Figures $j \cdot i)$. DID is not better than the other methods because of the extra $3 \mathrm{db}$ penalty.

4. Additive detection performs better in all cases, especially when all sources of jitter are important or when bit shuft jitter is not negligible (see Figures $5-i$ ).

\section{Conclusions}

This paper has introduced a jitter model and a quantization approach. based on which signal processing techniques in equal- ization. detection, and modulation were used to obtan beter dati recovery. From the simulation examples Illustrated. We found that additive detection plus velocity and ISI cqualizations betiorm: best. Its smailer SiVR to obtain the same matk error fro unumiesmailer sizes can be recovered and consequently a inginer densit. can be achieved.

\section{Acknowledgement}

The author would like to thank M. Mansuripur and R. Ilayjar of the Optical Sciences Center of the Linversity of Arizona. Davici Cheng and Roger Wood of IBM, and the anonymous reviewers ior their valuable help and input to this paper.

\section{References}

(1) Alan B. Marchant, Optical Recording, A Technical Overvieu Addision Wesley, 1990.

[2] Roger Wood. "Magnetic and Optical Storage systems: Opportunities for Communications Technology . pp. 1605-16:2. Proceeding of ICC. 1989.

(3) Dennis G. Howe. "Signal-to-nolse ratıo (SNR) for reituvit data recording", SPIE Vol. 695. Opucai Mass Data Storar. II, pp. 255-261. 1986.

[4] H. Burkhardt. "Phase Detection with Run-Length-Limited Codes", IBM Technical Disclosure Bulletin. Val. 24. No. 1B p. 683, June 1981 .

[5] B. P. Lathi. Chapter 3 of Modern Digutal and Analog Communication Systems.. 2nd Ed.. Holt. Rinehart, and Winsion. 1989.

[6] David C. Cheng. Private Communications. IBM Research Division. San Jose.

[7] T. W. McDaniel. "Simulation of bit jitter in magneto-oplic recording", J. of Appl. Phys., April 1988, pp. 3859-3861

[8] Max M.-K. Liu, "Experiments for Future High Density Ontical Data Storage", Paper presented in Optical Data Storage meeting, University of Arizona. Tucson. Apr. 1990. 
